\title{
XMM-Newton observations of Extremely Red Objects and the link with luminous, X-ray obscured quasars *
}

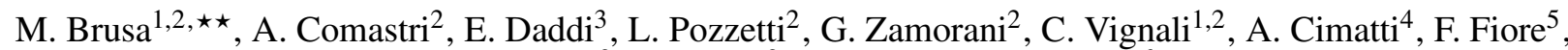 \\ M. Mignoli ${ }^{2}$, P. Ciliegi ${ }^{2}$, and H. J. A. Röttgering ${ }^{6}$ \\ 1 Dipartimento di Astronomia Universita' di Bologna, via Ranzani 1, 40127 Bologna, Italy \\ e-mail: marcella@mpe.mpg.de \\ 2 INAF - Osservatorio Astronomico di Bologna, via Ranzani 1, 40127 Bologna, Italy \\ 3 European Southern Observatory, Karl-Schwarzschild-Strasse 2, 85748 Garching bei Muenchen, Germany \\ 4 INAF - Osservatorio Astrofisico di Arcetri, Largo E. Fermi 5, 50125 Firenze, Italy \\ 5 INAF - Osservatorio Astronomico di Roma, via Frascati 33, 00040 Monteporzio, Italy \\ ${ }^{6}$ Sterrewacht Leiden, PO Box 9513, 2300 RA, Leiden, The Netherlands
}

Received 14 June 2004 / Accepted 28 October 2004

\begin{abstract}
We present the results of a deep (about $80 \mathrm{ks}$ ) XMM-Newton survey of the largest sample of near-infrared selected Extremely Red Objects $(R-K>5)$ available to date to $K_{\mathrm{S}} \lesssim 19.2$. At the relatively bright X-ray fluxes $\left(F_{2-10 \mathrm{keV}} \gtrsim\right.$ $4 \times 10^{-15} \mathrm{erg} \mathrm{cm}^{-2} \mathrm{~s}^{-1}$ ) and near-infrared magnitude probed by the present observations, the fraction of AGN (i.e. X-ray detected) among the ERO population is small $(\sim 3.5 \%)$; conversely, the fraction of EROs among hard X-ray selected sources is much higher $(\sim 20 \%)$. The X-ray properties of EROs detected in our XMM-Newton observation indicate absorption in excess of $10^{22} \mathrm{~cm}^{-2}$ in a large fraction of them. We have also considered additional samples of X-ray detected EROs available in the literature. X-ray spectral analysis of the highest $\mathrm{S} / \mathrm{N}$ sources unambiguously indicates that large columns of cold gas (even $>10^{23} \mathrm{~cm}^{-2}$ ) are the rule rather than the exception. The X-ray, optical and near-infrared properties of those X-ray selected EROs with a spectroscopic or photometric redshift nicely match those expected for type 2 quasars, the high-luminosity, high-redshift obscured AGNs predicted in baseline XRB synthesis models. A close correlation is detected between $X$ - and $K$-band fluxes. For the AGN EROs this is consistent, under reasonable assumptions, with the relation established locally between the host galaxies and their central black holes. This suggests that the majority of EROs are powered by massive black holes accreting, on average, at about $0.03-0.1$ of the Eddington limit.
\end{abstract}

Key words. X-rays: general - galaxies: active - galaxies: high-redshift - cosmology: observations - X-rays: galaxies

\section{Introduction}

Extremely Red Objects (EROs, $R-K>5$, Elston et al. 1988), initially detected in near-infrared ground-based imaging, have the colors expected for high-redshift passive ellipticals and have been used as tracers of distant $(z \gtrsim 1)$ and old spheroids. Reproducing their observational properties have proved to be extremely challenging for all current galaxy formation models (see e.g. Kauffmann 2003 for a review). However, on the basis of a number of observational results, it has been pointed out that high-redshift passive ellipticals are only one of the various classes of extragalactic sources which make up the ERO population. Deep VLT spectroscopy from the $K 20$ survey (Cimatti et al. 2002, 2003) has indeed shown that EROs are nearly

$\star$ Tables 1 and 2 are only available in electronic form at the CDS via anonymous ftp to cdsarc.u-strasbg.fr (130.79.128.5) or via http://cdsweb.u-strasbg.fr/cgi-bin/qcat?]/A+A/432/69

$\star \star$ Present address: Max Planck Institut fuer Extraterrestrische Physik, 85478 Garching, Germany. equally populated by old, passively evolving systems and dusty star-forming galaxies over a similar range of redshift $(z=$ 0.8-2 for both the classes; see also Yan et al. 2004) and similar results are confirmed both by colour selection criteria (Mannucci et al. 2002) and by radio observations (Smail et al. 2002). A few individual objects have been also identified as high redshift Active Galactic Nuclei (AGN) on the basis of the detection of strong emission lines in near-infrared and/or optical spectra (see e.g. Pierre et al. 2001; Brusa et al. 2003). In this case the enhanced emission in the $K$ band with respect to the $R$ band is probably due to the combination of strong dust extinction in the optical and a contribution of the point-like emission in the near-infrared. However, there is increasing evidence that the near-infrared light of obscured AGN is dominated by the host galaxy emission (see e.g. Mainieri et al. 2002; Mignoli et al. 2004).

A large population of optically faint X-ray sources without any obvious AGN signature in the optical spectrum and with optical to near-infrared colors typical of high redshift ellipticals 
and starburst galaxies has been revealed in the deepest Chandra and XMM-Newton exposures (e.g. Hasinger et al. 2001; Barger et al. 2003; Szokoly et al. 2004). Thus, the follow-up campaigns of deep XMM-Newton and Chandra observations have proved to be a powerful tool to investigate the AGN ERO population (Alexander et al. 2002).

Results from both shallow and deep X-ray surveys also suggested that the AGN population among EROs shares the same X-ray properties of high-luminosity, highly obscured $\left(N_{\mathrm{H}}>\right.$ $10^{22} \mathrm{~cm}^{-2}$ ) AGN (Mainieri et al. 2002; Alexander et al. 2002; Brusa 2003). Further support for the result that a significant fraction of obscured AGN are hosted in EROs comes from near infrared observations of X-ray sources selected on the basis of their high X-ray to optical flux ratio $(\mathrm{X} / O>10$, Mignoli et al. 2004): the hosts of luminous, obscured hard $X$-ray sources with extreme $\mathrm{X} / O$ are among the most massive spheroids at $z \gtrsim 1$.

Finally, the observed fraction of AGN among EROs can help constrain models which include the evolution of QSO activity in the formation of spheroids and the resulting effects on galaxy evolution (e.g. Granato et al. 2004; Menci et al. 2004). Several physical models have been proposed in which the fueling of the supermassive accreting black holes in AGN is triggered by merging events (in the context of the hierarchical structure formation paradigm), and the interplay between star formation and nuclear activity determines the relationship between the black hole $(\mathrm{BH})$ mass and the mass of the host galaxy. If the evolution of luminous AGN follows that of spheroids, as suggested by e.g. Franceschini et al. (1999) and Granato et al. (2001), it is possible that the radiation and the strong winds produced by a powerful AGN present in the massive galaxy may help inhibit the star-formation in these galaxies, which therefore would have red colors. However, previous studies on the fraction of AGN among the ERO population, although having deep near-infrared and X-ray observations $(K \sim 21-22$ and the Megaseconds Chandra exposures) were limited in area coverage (50-80 $\left.\operatorname{arcmin}^{2}\right)$ and therefore were unsuitable for detailed statistical analyses of the AGN EROs population.

To further study the nature of AGN EROs and the link between accreting supermassive black holes and the host galaxy properties, we have started an extensive program of multiwavelength observations of one of the largest samples of nearinfrared selected EROs available to date ( $\sim 400$ sources), selected over a contiguous field of $\sim 700 \operatorname{arcmin}^{2}$ (the "Daddi field", Daddi et al. 2000). The sample is complete to a magnitude limit of $K \mathrm{~s} \sim 19$ and the field is covered by deep optical photometry in the $R$-band. The same field will also be imaged with Subaru and Chandra and spectroscopic VIMOS observations are already planned. We have obtained with XMM-Newton a total of $110 \mathrm{ks}$, in two different observations: the moderate-deep exposure and the high energy throughput of XMM-Newton, coupled with its large field of view, are wellsuited to detect AGN among EROs at relatively bright X-ray fluxes, on a statistically significant sample. The data reduction and analysis, the X-ray source identification and the X-ray properties of X-ray detected EROs in our XMM-Newton sample are presented in Sect. 2. The results on the fraction of AGN EROs as a function of the X-ray and $K$-band fluxes are discussed in Sect. 3. Section 4 compares the optical, near infrared and X-ray properties of the EROs in our sample with those of other samples of X-ray detected EROs and discusses the fraction of AGN EROs in $K$-selected samples. Section 5 presents the average X-ray properties of EROs AGN, their contribution to the quasar 2 population and an estimate of their Black Hole masses and Eddington ratios. Section 6 summarizes the most important results. Throughout the paper, a cosmology with $H_{0}=70 \mathrm{~km} \mathrm{~s}^{-1} \mathrm{Mpc}^{-1}, \Omega_{\mathrm{m}}=0.3$ and $\Omega_{\Lambda}=0.7$ is adopted.

\section{Multiwavelength data and X-ray source identification}

\subsection{Near-infrared and optical data}

The near infrared ERO sample was selected by Daddi et al. (2000) from the $5 \sigma K$-band source catalog adopting the selection criterion $R-K \mathrm{~s} \geq 5$. The $R$-band data were taken at the $4.2 \mathrm{~m}$ William Herschel Telescope on La Palma, while the $K$ s observations were performed with the ESO NTT 3.5 m telescope in La Silla; relevant details on optical and near-infrared data reduction can be found in Daddi et al. (2000).

A total of 400 EROs are included in this "reference ERO sample"; to date, this study still constitutes the largest published survey of EROs performed at moderately deep $K$ limits, complete to $K \mathrm{~s}=18.8$ over $\sim 700 \mathrm{arcmin}^{2}$ and to $K \mathrm{~s}=19.2$ in a deeper area of $\sim 450 \mathrm{arcmin}^{2}$, more than a factor of four larger than other near-infrared surveys at the same limiting magnitudes (e.g. Thompson et al. 1999; Miyazaki et al. 2003). The $5 \sigma$ limiting magnitude in the $R$ band is $\sim 25.5$.

\subsection{X-ray data}

Two XMM-Newton observations of this field have been obtained with the European Photon Imaging Camera (EPIC, Jansen et al. 2001), equipped with both the $M O S$ and $p n$ instruments. The first observation (OBS-ID 0057560301) was taken on August 3, 2001 for a nominal exposure time of $50 \mathrm{ks}$; the second observation was taken two years later, in two parts (August 22, 2003 - OBS-ID 0148520101 - and September 16, 2003 - OBS-ID 0148520301), for a total nominal exposure time of $\sim 60 \mathrm{ks}$.

All the EPIC cameras operated in full-frame and were equipped with the "Thin" filter, which is usually employed in the observations of faint sources ${ }^{1}$. The three XMM-Newton datasets were reduced using version 5.4.1 of the Science Analysis System $^{2}$ (SAS) with the latest relevant calibration products. The raw $p n$ and MOS Observation Data Files (ODF) were processed using the SAS tasks emproc and epproc to produce calibrated event lists. Only events with pattern 0-4 (single and double) for the $p n$ and 0-12 for the MOS cameras were selected. All the event files were cleaned of hot pixels and soft proton flares removing all the time intervals with a count

\footnotetext{
1 http://xmm.vilspa.esa.es/external/ xmm_user_support/documentation/index.shtml

2 http://xmm.vilspa.esa.es/external/ xmm_sw_cal/sas_frame.shtml
} 


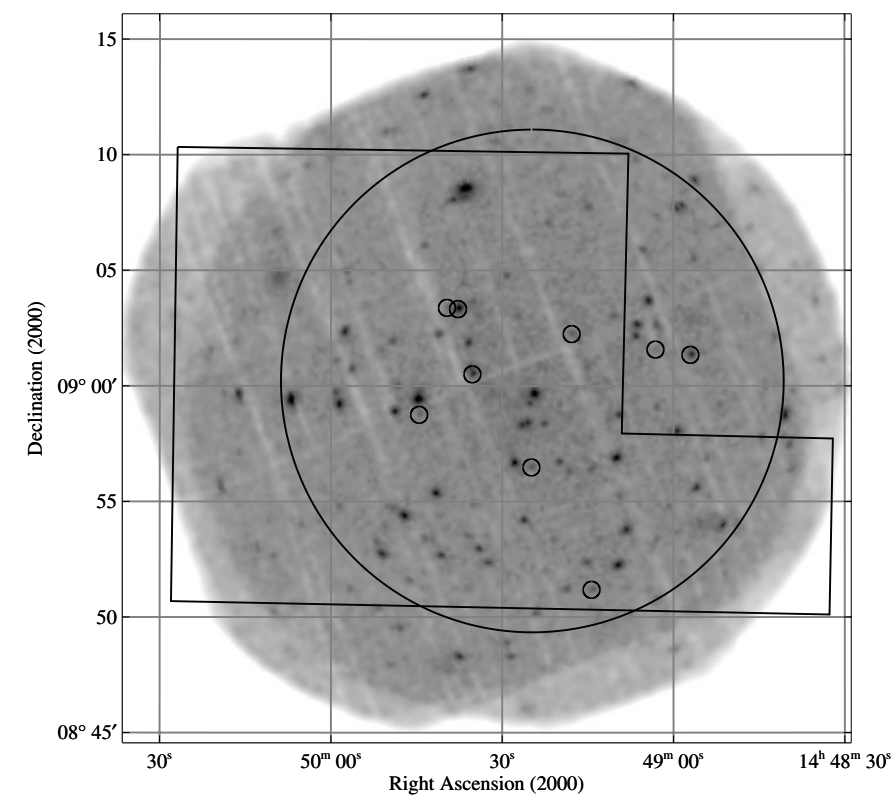

Fig. 1. X-ray image in the $0.5-10 \mathrm{keV}$ band (grey scale) with the 11 arcmin radius circle of the $\mathrm{X}$-ray analysis and the deepest region $\left(\sim 450 \mathrm{arcmin}^{2}\right)$ in the $K$-band (polygon) superimposed. The shallower $K$-band image covers the entire $\mathrm{X}$-ray field of view. The $\mathrm{X}$-ray sources associated with EROs are marked with circles.

rate higher than $0.15 \mathrm{c} / \mathrm{s}$ in the $10-12.4 \mathrm{keV}$ energy range for the MOS and higher than $0.35 \mathrm{c} / \mathrm{s}$ in the $10-13 \mathrm{keV}$ band for the $p n$ units (see Baldi et al. 2002).

The excellent relative astrometry between the three cameras in each observation (within $1^{\prime \prime}$, well below their PSF $F W H M$ of $\sim 6^{\prime \prime}$ ) allowed us to merge the MOS and $p n$ images in each observation, thus increasing the signal-to-noise ratio and reaching fainter X-ray fluxes. Moreover, taking into account the absolute astrometry between the three observations, the counting statistics have been improved by summing all the available datasets (i.e. MOS1, MOS2 and pn of the three observations). The resulting total exposure time for the $p n$ is $\sim 82 \mathrm{ks}$, and is only slightly lower for the $M O S$ instruments ( $\sim 78 \mathrm{ks})$.

We have limited the X-ray analysis to a circular region of 11 arcmin radius from the point of maximum exposure time (centered at $\alpha=14^{\mathrm{h}} 49^{\mathrm{m}} 25^{\mathrm{s}}$ and $\delta=09^{\circ} 00^{\prime} 13^{\prime \prime}$ ). At this radius, the effective exposure drops to $\sim 50 \%$ of the maximum value. The resulting area analysed in the present work is therefore of $\sim 380 \mathrm{arcmin}^{2}$. The area in common with the deepest nearinfrared coverage is $\sim 300 \mathrm{arcmin}^{2}$. The pattern of the X-ray and optical/near-infrared data is shown in Fig. 1: the 11 arcmin radius circle and the deepest $K_{\mathrm{s}}$ band region (polygon) are superimposed on the $0.5-10 \mathrm{keV}$ image.

The XMM-Newton area analysed in this work includes 257 EROs: 173 EROs with $K \mathrm{~s} \lesssim 18.8$ over $\sim 380 \mathrm{arcmin}^{2}$ and 216 EROs with $K \mathrm{~s} \lesssim 19.2$ in the area with the deeper nearinfrared coverage $\left(\sim 300 \mathrm{arcmin}^{2}\right)$. The accurate detection algorithm developed for the Hellas $2 \mathrm{Xmm}$ survey (see Baldi et al. 2002 for details) was run on the $0.5-10 \mathrm{keV}$ (full band) cleaned events, in order to create a preliminary list of candidate sources which included also sources detected at a low level of significance. We then computed for each source the probability that the detected counts originate from Poissonian background fluctuations and we kept in the final list only those sources (96) which were detected above a detection probability threshold of $p=2 \times 10^{-5}$ (that corresponds to less than 1 spurious X-ray detection, or $\simeq 4 \sigma$ Gaussian threshold). The count rate to flux conversion factor was derived assuming a power law with photon index $\Gamma=1.7$, absorbed by the Galactic column density in the direction of the Daddi Field $\left(N_{\mathrm{H}}=5 \times 10^{20} \mathrm{~cm}^{-2}\right.$, Dickey \& Lockman 1990), and weighted by the effective exposure times of the different EPIC cameras. The uncertainty in the derived fluxes is $<15 \%$ for $\Delta \Gamma= \pm 0.5$ and $N_{\mathrm{H}}$ up to $10^{21} \mathrm{~cm}^{-2}$. The corresponding full band limiting flux is $\sim 2 \times 10^{-15} \mathrm{erg} \mathrm{cm}^{-2} \mathrm{~s}^{-1}$ at the aim point, and is a factor of $\sim 1.5$ higher at the edge of the selected area, where the net exposure is $\sim 40 \mathrm{ks}$.

In order to compute reliable X-ray fluxes in different energy bands (i.e. to roughly account for the X-ray spectral shape), fluxes in the soft $(0.5-2 \mathrm{keV}$ band) and hard (2-10 keV) band were computed from the counts detected in each energy band using the same detection algorithm. A total of 86 sources (down to $\left.S_{\lim } \sim 9 \times 10^{-16} \mathrm{erg} \mathrm{cm}^{-2} \mathrm{~s}^{-1}\right)$ and 60 sources $\left(S_{\lim } \sim 4 \times\right.$ $10^{-15} \mathrm{erg} \mathrm{cm}^{-2} \mathrm{~s}^{-1}$ ) were detected, respectively.

The X-ray centroids have been astrometrically calibrated with respect to the optical positions of three bright quasars in the field (Hall et al. 2000): the resulting shift of $\sim 2^{\prime \prime}(\Delta(\mathrm{RA})=$ $\left.1.47^{\prime \prime} ; \Delta(\mathrm{Dec})=-1.29^{\prime \prime}\right)$ was then applied to all of the source positions.

Table 1 lists all the relevant X-ray properties of the detected sources (the X-ray source identifier, X-ray coordinates, hard $2-10 \mathrm{keV}$ flux and counts, soft $0.5-2 \mathrm{keV}$ flux and counts), sorted with decreasing hard band X-ray flux.

\subsection{Likelihood analysis}

Optical and near-infrared photometry is available for 87 (55) out of 96 (60) X-ray sources detected in the full (hard) band. The sources for which optical and near-infrared photometry is not available are all close to bright stars and/or defects in the $R$ and/or $K \mathrm{~s}$ band images that were masked in the optical and near-infrared source detection.

At the optical and near-infrared fluxes probed by our survey, the identification process is a critical issue, especially for faint sources. At first, the X-ray source list was cross-correlated with the $K$-band and $R$-band catalogs using a conservative $5^{\prime \prime}$ radius error circle (see Brusa et al. 2003 for further details): 162 (71) sources in the $R(K \mathrm{~s})$ band were found in $87 \mathrm{X}$-ray error-circles. The difference in the number of objects (i.e. surface densities) in the two bands clearly reflects the different depth of the optical and near-infrared images. In particular, using the $R$ catalog and a fixed search radius, substantial source confusion may be present: on the basis of the integral counts from the $R$-band catalog, on average 1 galaxy with $R<25$ is expected by chance in each of the $5^{\prime \prime}$ radius error circles. We therefore decided to use the "likelihood ratio" $(L R)$ technique, in order to properly identify the optical/near-infrared counterparts. The $L R$ is defined as the ratio between the probability that the source is the correct identification and the corresponding 
probability of being a background, unrelated object (Sutherland \& Saunders 1992), i.e.:

$L R=\frac{q(m) f(r)}{n(m)}$

where $f(r)$ is the probability distribution function of the positional errors and it is assumed to be a two-dimensional Gaussian, $n(m)$ is the surface density of background objects with magnitude $m$, and $q(m)$ is the expected probability distribution as a function of magnitude of the true counterparts. The $q(m)$ distribution is normalized as $\int^{m_{\text {faint }}} q(m) \mathrm{d} m=Q$, where $Q$ is the a priori expected fraction of X-ray sources with an optical counterpart brighter than $m_{\text {faint }} ; m_{\text {faint }}$ can be either the limiting magnitude of the optical data or the magnitude beyond which the surface density of background objects becomes so high that no reliable "statistical" identification is possible.

For the calculation of the $L R$ parameters we have followed the procedure described by Ciliegi et al. (2003); more specifically, in order to maximize the statistical significance of the overdensity due to the presence of the optical counterparts, we have adopted a $3^{\prime \prime}$ radius for the estimate of the $q(\mathrm{~m})$ distribution. A large fraction of the possible counterparts are expected to be included within such a radius, on the basis of previous works on XMM-Newton data (e.g. Fiore et al. 2003).

Figure 2 shows the observed magnitude distribution of the optical objects detected in the $R$ band within a radius of $3^{\prime \prime}$ around each X-ray source (solid histogram), together with the expected distribution of background objects in the same area (dashed histogram). The difference between these two distributions (dot-dot-dot dashed histogram) is the expected magnitude distribution of the optical counterparts. The smooth curve fitted to this histogram (dot-dot-dot dashed line) has been used as the input in the likelihood calculation $(q(m))$. Figure 2 shows that the observed number of objects is well above the background up to $R \sim 24$. At $R>24$, the number of detected objects in the X-ray error boxes is consistent with that expected from the background. For these reasons we have adopted $R_{\text {faint }} \sim 24$ in our likelihood calculation; all sources fainter than this limit will have $q(m)=0$ by definition and correspondingly $L R=0$. For the $Q$ normalization we adopted $Q=0.75$, corresponding to the ratio between the integral of the $q(m)$ ) distribution and the total number of X-ray sources. This preliminary analysis suggests that we expect to identify a fraction of the order of $75 \%$ of the X-ray sources with objects brighter than $R \sim 24$, in agreement with the results from other XMM-Newton surveys (e.g. Hasinger et al. 2001). A similar procedure has been applied to the $K$ band data and the $L R$ value for all the optical and nearinfrared candidates has been computed.

The next step is to choose the best threshold value for $L R\left(L_{\mathrm{th}}\right)$ to discriminate between spurious and real identifications. The choice of $L_{\text {th }}$ depends on two factors: first, it should be small enough to avoid missing many real identifications and producing a rather incomplete sample. Secondly, $L_{\text {th }}$ should be large enough to keep the number of spurious identifications as low as possible and to increase the reliability of the source identifications. An $L R$ threshold of $L_{\text {th }}=0.25$ in both optical and near-infrared bands has been adopted; this turned out to be the value that maximizes the sum of sample

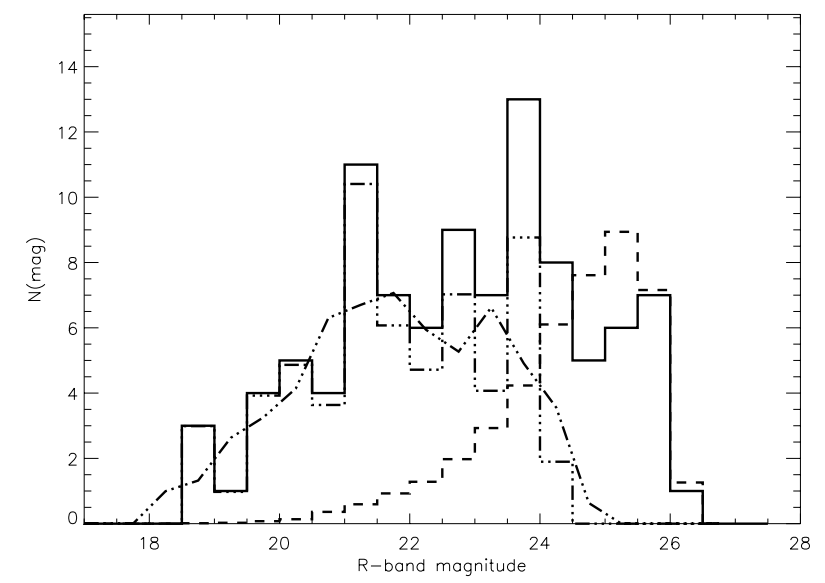

Fig. 2. Observed magnitude distribution of the optical objects detected in the $R$ band within a radius of $3^{\prime \prime}$ around each of the $87 \mathrm{X}$-ray sources (solid histogram), together with the expected distribution of background objects in the same area $(n(m)$, dashed histogram). The difference between these two distributions (dot-dot-dot dashed histogram) is the expected magnitude distribution of the optical counterparts. The smooth curve fitted to this histogram (dot-dot-dot dashed line) has been used as input in the likelihood calculation $(q(m))$.

reliability and completeness for the assumed $Q$ normalization (see Ciliegi et al. 2003 for further details). To check how the uncertainty in $Q$ could affect our results, we repeated the likelihood ratio analysis using different values of $Q$ in the range 0.5-1.0: no substantial difference in the final number of identifications was found.

This threshold, a posteriori, led to an estimated percentage of secure X-ray to optical or near-infrared associations up to $m_{\text {faint }}$ of the order of $\sim 80 \%$, in good agreement with the estimate of $Q$.

\subsection{X-ray source identification}

The information derived in the two bands were then combined: all the sources with the highest $L R>L_{\text {th }}$ in both the $R$ and $K$ bands, as well as the sources undetected in the $K$ band but with a $L R>L_{\text {th }}$ in the $R$-band were defined as secure identifications (a total of 70). As expected, most of the reliable optical counterparts have an X-ray to optical separation $(\Delta(\mathrm{X}-O))$ smaller than $3^{\prime \prime}$, with only 4 objects with $3.16^{\prime \prime}<\Delta(\mathrm{X}-O)<3.71^{\prime \prime}$.

In addition, three $\mathrm{X}$-ray sources have a unique, very faint (24.5 $\lesssim \lesssim 25.2$ ) optical counterpart within 1.6" from the X-ray position (xid\#330, xid\#244, xid\#129) ${ }^{3}$; by construction (see Sect. 2.3), their associated likelihood ratio is zero. Given that less than one galaxy with $24 \lesssim R \lesssim 25$ is expected by chance in the total area corresponding to 87 error boxes with a $1.6^{\prime \prime}$ error-box, we tentatively consider also these three sources as identifications. Seventy-three out of $87 \mathrm{X}$-ray sources have been therefore securely associated with an optical/near-infrared counterpart and are reported in the first part of Table 2, where we list, for each X-ray source, the X-ray ID number and

3 These sources have no further optical counterparts within an errorbox of 5" radius. 
Table 3. Hard X-ray detected EROs.

\begin{tabular}{|c|c|c|c|c|c|c|c|c|c|c|c|}
\hline ID & $\begin{array}{l}\mathrm{RA}(\mathrm{X}) \\
(\mathrm{J} 2000)\end{array}$ & $\begin{array}{l}\operatorname{Dec}(\mathrm{X}) \\
(\mathrm{J} 2000)\end{array}$ & $\begin{array}{c}\Delta(\mathrm{X}-O) \\
" \prime\end{array}$ & $R\left(2^{\prime \prime}\right)$ & $K\left(2^{\prime \prime}\right)$ & $R-K$ & $\begin{array}{c}F_{2-10 \mathrm{keV}} \\
\operatorname{erg~cm}^{-2} \mathrm{~s}^{-1}\end{array}$ & $H R$ & $\log (\mathrm{X} / O)$ & $\log (\mathrm{X} / K)$ & $L R(K)$ \\
\hline \multicolumn{12}{|c|}{$5 \sigma$ ERO sample } \\
\hline 148 & 222.40681 & 9.05575 & 0.37 & 25.24 & 18.76 & 6.48 & $2.9 \mathrm{e}-14$ & -0.44 & 2.06 & 0.97 & 15.78 \\
\hline 195 & 222.23720 & 9.02275 & 1.73 & 24.11 & 18.32 & 5.79 & $2.5 \mathrm{e}-14$ & 0.00 & 1.54 & 0.73 & 15.58 \\
\hline 209 & 222.39636 & 9.00872 & 0.64 & 23.27 & 18.08 & 5.19 & $9.5 e-15$ & -0.54 & 0.78 & 0.21 & 22.96 \\
\hline 293 & 222.35330 & 8.94158 & 2.81 & 24.26 & 19.13 & 5.13 & $9.1 \mathrm{e}-15$ & $>0.76$ & 1.16 & 0.61 & 4.92 \\
\hline 239 & 222.30940 & 8.85337 & 0.40 & 23.96 & 18.23 & 5.73 & $8.9 e-15$ & -0.19 & 1.03 & 0.24 & 23.84 \\
\hline 146 & 222.41489 & 9.05666 & 2.31 & 25.63 & 19.10 & 6.53 & $6.5 e-15$ & 0.01 & 1.56 & 0.45 & 7.23 \\
\hline 189 & 222.26312 & 9.02621 & 2.02 & 24.40 & 19.04 & 5.36 & $5.8 \mathrm{e}-15$ & $>0.32$ & 1.02 & 0.38 & 8.73 \\
\hline 250 & 222.43523 & 8.97954 & 1.36 & 23.50 & 18.47 & 5.03 & $5.8 \mathrm{e}-15$ & $>0.42$ & 0.66 & 0.15 & 14.69 \\
\hline \multicolumn{12}{|c|}{$3 \sigma$ ERO sample } \\
\hline 175 & 222.32430 & 9.03741 & 1.44 & 24.39 & 19.35 & 5.04 & $7.9 e-15$ & 0.12 & 1.24 & 0.43 & - \\
\hline
\end{tabular}

position, the distance between the X-ray and optical counterparts (or candidates), the $R$ and $K$-band magnitudes, and the $L R$ in both the optical and near-infrared bands. Of these 73 sources, 45 belong to the hard X-ray sample. In an other 8 cases we have more than one possible optical and/or near-infrared counterpart with $L R>L R_{\text {th }}$ and therefore the real counterparts are not unambiguously determined. These are listed in the second part of Table 2 In these cases, a detection in the forthcoming Chandra observations (with 5-10 times smaller errorboxes) would definitively discriminate between the possible counterparts.

In the remaining 6 cases the possible counterparts have on average fainter optical magnitude, none of the candidates has $L R>L_{\mathrm{th}}$, and all of them lie at $\Delta(\mathrm{X}-O)>3^{\prime \prime}$ (Table 2). This can be the case if the source is very faint and undetected in the optical bands (see e.g. Koekemoer et al. 2004), or if the $\mathrm{X}$-ray emission originates from a group of galaxies. In the bottom part of Table 2 we list the 9 X-ray sources for which optical and infrared photometry is not available. In the following, we will consider only the ERO sources; a more detailed discussion of the properties of the global sample of optical/near-infrared identifications will be presented elsewhere (Brusa et al., in preparation).

\subsection{Hard X-ray detected EROs and hardness ratio analysis}

From the likelihood analysis, 8 hard X-ray sources are securely associated with EROs in the "reference ERO sample" (i.e., in the $5 \sigma$ catalog, see Sect. 2.1). All the EROs associated with $\mathrm{X}$-ray sources are reported in Table 3. Differently from Table 2, the $R$ and $K$ magnitudes given here are measured within a $2^{\prime \prime}$ diameter aperture. These are the magnitudes used to compute the $R-K$ colour and to select the ERO sample. The same table gives, for each source, the hardness ratio (HR) defined as $(\mathrm{H}-\mathrm{S}) /(\mathrm{H}+\mathrm{S})$ where $\mathrm{H}$ and $\mathrm{S}$ are the counts in the hard and soft

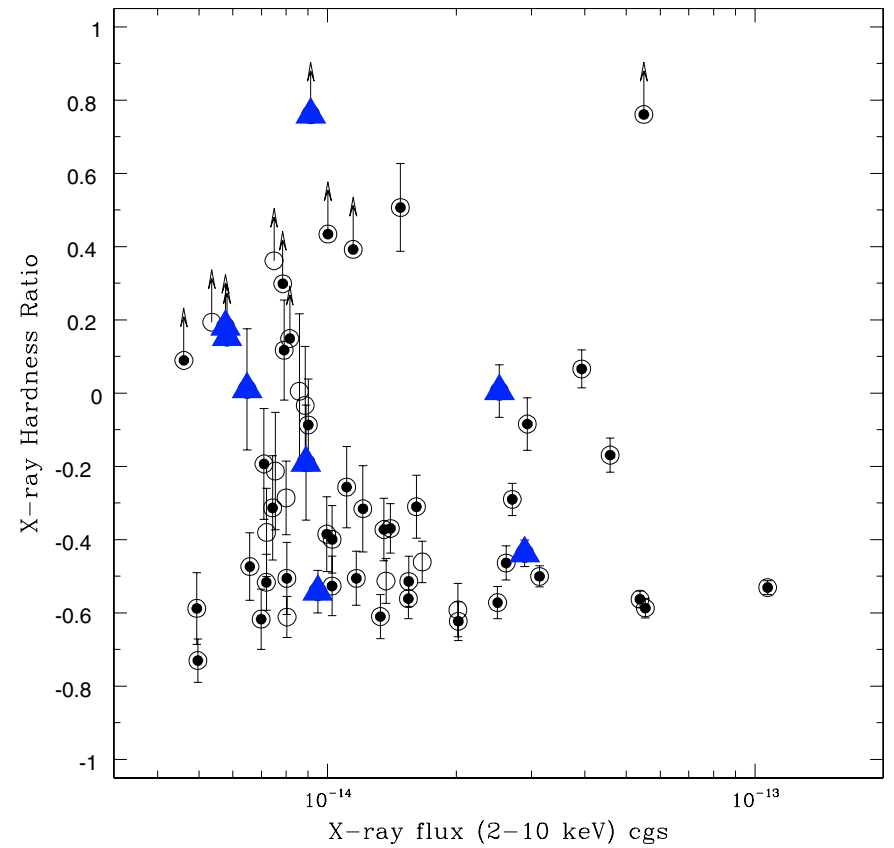

Fig. 3. The hardness ratio versus $2-10 \mathrm{keV}$ flux for the hard X-ray selected sources in the "Daddi Field". Sources with a secure optical ID (i.e. first part of Table 1) are indicated as filled symbols. The 8 hard $\mathrm{X}$-ray detected EROs from the $5 \sigma$ sample are plotted as filled triangles (see Table 3).

band, respectively. Among the $8 \mathrm{X}$-ray detected EROs, 5 are detected in both the hard and soft bands and three only in the hard (lower limit to HR).

One more X-ray source is associated with an ERO with a $K$ magnitude fainter than the $5 \sigma$ threshold (bottom part of Table 3). A total of 9 hard X-ray sources are therefore associated with EROs.

Figure 3 shows the HR versus the $2-10 \mathrm{keV}$ flux for the 60 hard X-ray selected sources. The eight EROs belonging to the 5 sigma sample are plotted as filled triangles. Six out of 
Table 4. Fraction of Hard X-ray detected EROs.

\begin{tabular}{|c|c|c|c|c|c|}
\hline$K(\lim )$ & $\begin{array}{c}F_{2-10 \mathrm{keV}}(\lim ) \\
\operatorname{erg~cm}^{-2} \mathrm{~s}^{-1}\end{array}$ & $\begin{array}{c}\text { area } \\
\operatorname{arcmin}^{2}\end{array}$ & $\begin{array}{c}N(\text { EROs }) \\
K \text {-selected }\end{array}$ & $\begin{array}{c}N(\text { EROs }) \\
\text { X-ray detected }\end{array}$ & $\%$ \\
\hline \multicolumn{6}{|l|}{ This work: } \\
\hline 18.8 & $4 \times 10^{-15}$ & $\sim 380$ & 173 & 6 & $3.5 \%$ \\
\hline 19.2 & $4 \times 10^{-15}$ & $\sim 300$ & 216 & 7 & $3.2 \%$ \\
\hline all sample & $4 \times 10^{-15}$ & $\sim 380$ & 257 & 9 & $3.5 \pm 1.2 \%$ \\
\hline \multicolumn{6}{|l|}{$\mathrm{A} 02^{\dagger}:$} \\
\hline 20.1 & $2 \times 10^{-16}$ & $\sim 70$ & $29^{\ddagger}$ & 4 & $14_{-7}^{+11} \%$ \\
\hline \multicolumn{6}{|l|}{$\mathrm{R} 03^{\dagger}:$} \\
\hline 21.5 & $4 \times 10^{-16}$ & $\sim 50$ & 179 & 12 & $6.6_{-1.8}^{2.0} \%$ \\
\hline
\end{tabular}

: AO2: Alexander et al. (2002); R03: Roche et al. (2003).

Selected on the basis of a $I-K>4$ criterion.

eight EROs have HR values higher than the median value of the total sample $\left(\mathrm{HR}_{\text {med }}=-0.3\right)$; the HR of these 6 EROs imply $N_{\mathrm{H}}>10^{22} \mathrm{~cm}^{-2}$ at $z>1$ (see Fig. 7 in Brusa et al. 2003). Only two EROs have HR values in the range expected for unobscured AGN (HR -0.4), and they are detected at $F_{2-10 \mathrm{keV}} \gtrsim 10^{-14} \mathrm{erg} \mathrm{cm}^{-2} \mathrm{~s}^{-1}$, while the majority of the EROs are detected at $F_{2-10 \mathrm{keV}} \lesssim 10^{-14} \mathrm{erg} \mathrm{cm}^{-2} \mathrm{~s}^{-1}$ and hard X-ray colours, qualitatively in agreement with the observed hardening of the X-ray source population at fainter flux levels (e.g., Tozzi et al. 2001).

These data strongly suggest that significant absorption is present in a large fraction of the hard X-ray detected EROs population (see also Sect. 5). On the other hand, Fig. 3 shows that even if the fraction of EROs increases among sources with higher values of $H R$, not all of the hardest sources are EROs (e.g. among the 15 sources with $H R>0$, only 6 are EROs).

\section{Fractions of X-ray detected EROs and of EROs among hard X-ray sources}

The large area of our sample is well suited to statistically assess the fraction of AGN powered EROs at relatively bright X-ray fluxes and to quantitatively investigate the luminous tail of this population.

$257 \mathrm{~K}$-selected EROs from the "reference ERO sample" are within the XMM-Newton area analysed in this work. Among the 9 hard X-ray detected objects, seven are in the deeper $(K \mathrm{~s} \lesssim 19.2) 300 \operatorname{arcmin}^{2}$ (see Fig. 1), while the number of $\mathrm{X}$-ray detected EROs in the $380 \mathrm{arcmin}^{2}$ area at the shallower $K$ s $<18.8$ limit is 6; one additional object (xid\#189) is detected over the incomplete $18.8 \lesssim K \mathrm{~s} \lesssim 19.2$ area (see also Table 4 ).

In the present observation, the X-ray limiting flux corresponds to an X-ray luminosity $L_{\mathrm{X}} \gtrsim 10^{43} \mathrm{erg} \mathrm{s}^{-1}$ for $z=1$. Thus, the EROs X-ray emission is most likely powered by AGN activity, and the fraction of AGN among EROs in the present sample is at least $3.2 \pm 1.7 \%(8 / 257)$. The corresponding surface densities of X-ray emitting EROs are $\sim 1.6 \pm 0.6 \times 10^{-2} \operatorname{arcmin}^{-2}\left(6\right.$ over $\left.380 \operatorname{arcmin}^{2}\right)$ and $2.3 \pm 0.9 \times 10^{-2} \operatorname{arcmin}^{-2}\left(7\right.$ over $\left.300 \operatorname{arcmin}^{2}\right)$ at $K \mathrm{~s} \lesssim$ 18.8 and $K \mathrm{~s} \lesssim 19.2$, respectively.

The fraction of X-ray detected EROs in $K$-selected samples has been reported by Alexander et al. (2002; hereafter A02) and Roche et al. (2003; hereafter R03). A02 detected 6/29 EROs ${ }^{4}$ $(21 \%)$ in the Chandra Deep Field-North (CDF-N) observation, where the $K$-band limit is about one magnitude fainter ( $K \mathrm{~s}=20.1$ ) and the X-ray limiting flux is about one order of magnitude deeper than in our observation, with a corresponding limiting luminosity of $L_{\mathrm{X}} \simeq 10^{42} \mathrm{erg} \mathrm{cm}^{-2} \mathrm{~s}^{-1}$ at $z=1$. At the faint X-ray fluxes probed by ultra-deep Chandra exposures, starbursts and normal elliptical galaxies start to be detected and they are usually characterized by softer X-ray colors; a conservative estimate of the fraction of AGN powered EROs in the $\mathrm{CDF}-\mathrm{N}$ based on the hard X-ray detections and luminosities (see also Sect. 5) is $14_{-7}^{+11} \%$ (A02; Vignali et al. 2002).

From the R03 sample it is possible to estimate the fraction of hard X-ray detected EROs in the CDF-S GOODS area, at an $\mathrm{X}$-ray limiting flux comparable to that of the CDF-N sample but extending down to significantly fainter near-infrared magnitudes $(K \mathrm{~s} \sim 22)$ : about $6.6 \%$ of the $K$ s-selected EROs are associated with hard X-ray sources.

Even if the estimates from A02 and R03 at the faintest $K$ magnitudes are obtained over very small areas and may suffer from substantial cosmic variance, the differences in the fractions of X-ray detected EROs observed in these three samples are likely to be mainly due to the combination of different X-ray and near-infrared limiting fluxes, as discussed in Sect.4.3.

With XMM-Newton and Chandra surveys, the fraction of optical counterparts with extremely red colors has significantly increased with respect to the first examples of EROs found in deep ROSAT observations in the Lockman Hole (Lehmann et al. 2001). The present data imply that a fraction of the order of about $16-18 \%$ of the hard X-ray selected XMM-Newton

\footnotetext{
4 We note that these authors adopted an $I-K>4$ selection for the definition of their ERO sample, that roughly corresponds to a $R-K>$ 5.3 selection.
} 
sources exhibit $R-K>5$ colors (9/55 or 9/49 considering only the secure $\mathrm{X}$-ray to optical associations). Our results are in agreement with those reported by Mainieri et al. (2002) in the Lockman Hole: 12/53 ( 23\%) of hard X-ray selected sources are associated with EROs, at limiting near-infrared and X-ray fluxes comparable with those of the present sample. This fraction is about the same in the R03 sample $(\sim 23 \%)$ and in the CDF-N sample ( $21 \%$, from the Barger et al. 2003 catalog).

\section{Multiwavelength properties of AGN EROs}

\subsection{The "literature sample" of hard X-ray detected EROs}

To investigate the nature of hard X-ray selected EROs and the link between faint hard X-ray sources and the ERO population, we have collected all the literature multiwavelength data available to date for EROs individually detected in hard $(2-10 \mathrm{keV})$ $\mathrm{X}$-rays and selected on the basis of a $R-K>5$ criterion. More specifically:

1) Seventy EROs detected in the $2-8 \mathrm{keV}$ band in the CDF-N observation, from the Barger et al. (2003) catalog; 9 have spectroscopic redshifts and 25 have photometric redshift estimates;

2) twenty-two hard X-ray detected EROs in the Chandra Deep Field-South (CDF-S) observation, from Szokoly et al. (2004); 8 have spectroscopic redshift;

3) twelve EROs detected in the hard $(2-10 \mathrm{keV})$ band in the XMM-Newton Lockman Hole observation (Mainieri et al. 2002); 2 have spectroscopic redshifts and 3 have photometric redshift;

4) ten hard X-ray detected EROs from the Hellas2Xmm survey selected on the basis of an X-ray to optical flux ratio $\mathrm{X} / O>$ 10 and $R>24$; all of them with redshifts estimated on the basis of the observed $R-K$ colors (Mignoli et al. 2004);

5) five additional hard $X$-ray detected EROs available in the literature ("additional sample": Gandhi et al. 2004; Crawford et al. 2002; Brusa et al. 2003; Willott et al. 2003); 4 have spectroscopic redshifts and 1 photometric redshift.

This literature sample consists of 128 EROs, including the 9 EROs discussed in the present work, detected in the $2-10 \mathrm{keV}$ band; for 62 of them photometric or spectroscopic redshifts are available. This sample is by no means homogeneous and complete, but can be considered representative of EROs individually detected in X-rays.

\subsection{X-ray to optical/near-infrared properties of AGN EROs}

This enlarged ERO sample spans a wide range of optical and hard X-ray fluxes. The $R$-band magnitudes plotted versus the hard X-ray fluxes for all the 128 EROs in the literature sample are reported in Fig. 4 (upper panel): the 9 EROs from this work are reported as triangles, the 10 EROs from the Hellas2Xmm survey as squares and the remaining objects as circles. In the same figure, the sources classified as broad line (BL) AGN in
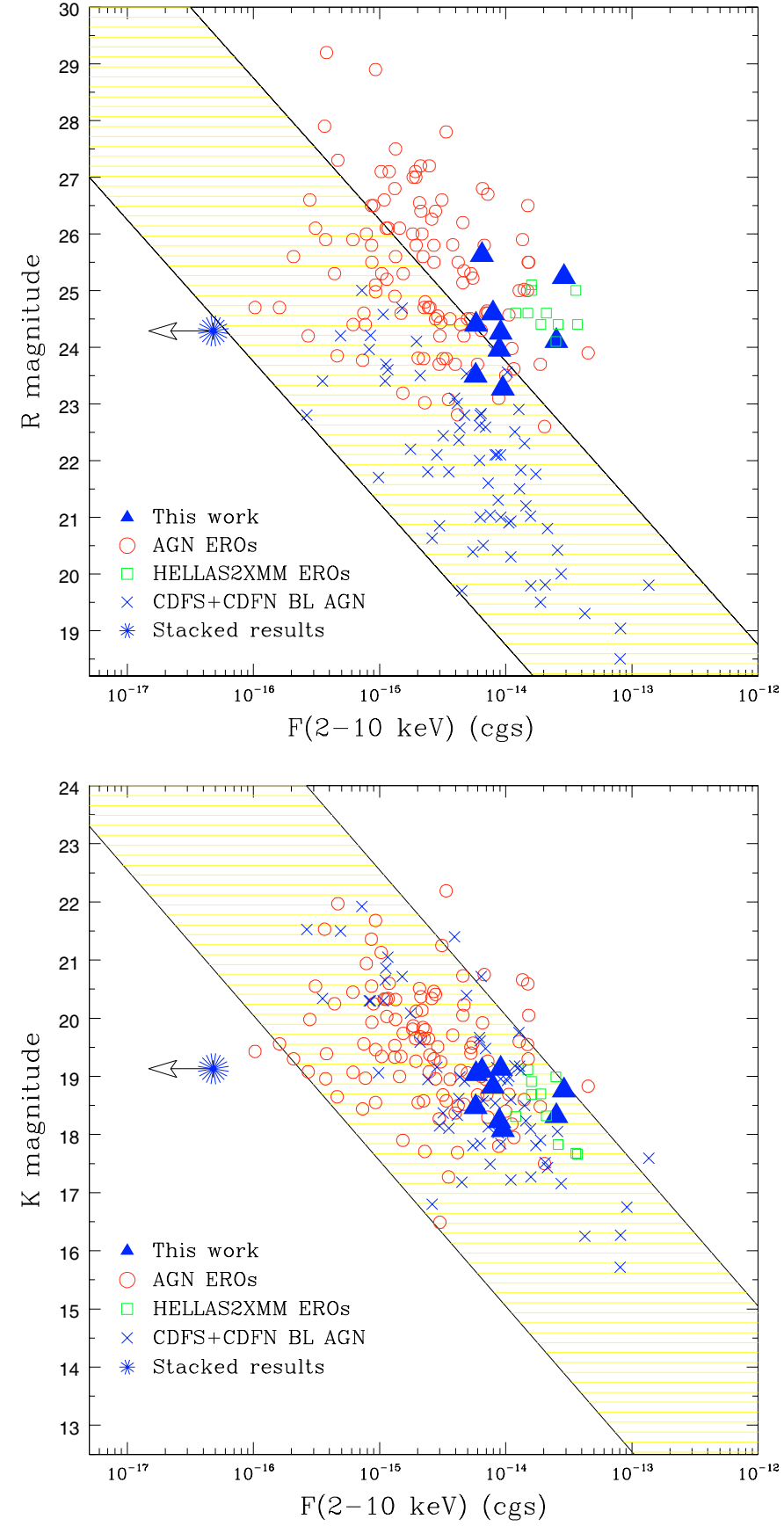

Fig. 4. Upper panel: $R$-band magnitude vs. hard X-ray flux for EROs, serendipitously detected in hard X-ray surveys. Large filled triangles are the 9 hard X-ray selected EROs of this work; circles correspond to the EROs in the "literature sample"; squares are sources from the Hellas2Xmm survey. As a comparison, broad-line AGN, i.e. sources with broad optical emission lines in the optical spectrum, detected in the $\mathrm{CDF}-\mathrm{S}$ and $\mathrm{CDF}-\mathrm{N}$ surveys are also reported as crosses. The shaded area represents the region typically occupied by known AGN (e.g. quasars and Seyferts) along the correlation $\log (\mathrm{X} / O)=0 \pm 1$. For comparison, we report the result of the stacking analysis performed on the K20 EROs in the CDF-S field not individually detected in the Chandra observation (asterisk at the faintest X-ray flux; Brusa et al. 2002). Lower panel: the same plot but in the $K$-band.

the CDF-N and CDF-S optical catalogs (Barger et al. 2003; Szokoly et al. 2004) are also reported as crosses. 
$\mathrm{X}$-ray detected EROs show an average ratio between the $\mathrm{X}$-ray and optical fluxes ${ }^{5}(\mathrm{X} / O)$ around $\mathrm{X} / O \simeq 10$, about one order of magnitude higher than that found for BL quasars by ROSAT (Hasinger et al. 1998; Lehmann et al. 2001) and recently extended by Chandra and XMM-Newton observations (Alexander et al. 2001; Rosati et al. 2002; crosses in Fig. 4). We note that the same shift with respect to the majority of quasar population is observed also excluding the 10 Hellas $2 \mathrm{Xmm}$ sources selected on the basis of their high $\mathrm{X} / O(>10)$.

The observed X-ray to optical properties of X-ray detected EROs are different to those of the majority of near-infrared selected EROs: the results of the stacking analysis of EROs not individually detected in the $\mathrm{X}$-rays in the $\mathrm{K} 20$ survey (asterisk in Fig. 4) led to an average $\mathrm{X} / O$ which is at least two order of magnitudes lower than that of the EROs in the present sample (Brusa et al. 2002; see also Alexander et al. 2002).

Obscured accretion at high redshifts is the most likely mechanism to explain the observed X-ray to optical properties. Moving the Spectral Energy Distribution (SED) of an X-ray absorbed AGN to progressively higher redshifts the K-corrections in the optical and X-ray band work in the opposite direction. The ratio between the optical to X-ray optical depth, in the observer frame, scales roughly as $(1+z)^{3.6}$, because dust extinction increases in the UV while X-ray absorption strongly decreases going toward high energies. The net result is that in the presence of an absorbing screen the observed optical flux of a high-z AGN can be strongly reduced, and the observed magnitudes are mainly due to starlight in the host galaxies. Conversely, the $2-10 \mathrm{keV} X$-ray flux can be much less reduced. Many extreme X-ray to optical ratio sources could then be highly obscured quasars, i.e. type 2 QSO (Fiore et al. 2003; Comastri et al. 2003). The observed high values of the $\mathrm{X} / O$ are therefore at least qualitatively consistent with those expected by a population of high redshift, absorbed AGN with X-ray column densities in the range $N_{\mathrm{H}}=10^{22}-10^{24} \mathrm{~cm}^{-2}$ (see also Sect. 5).

The lower panel of Fig. 4 shows that in the $K$-band magnitude vs. X-ray flux plane the X-ray detected EROs occupy essentially the same region as the broad-line AGN, with an average X-ray to near infrared ratio $(\mathrm{X} / K)$ of the order of 1 . Given that the $K$-band is less affected by absorption, the fact that AGN EROs are indistinguishable from the overall quasar population in this plane supports the hypothesis that their high $\mathrm{X} / O$ ratios are mainly due to significant nuclear extinction in the optical band (see also Mainieri et al. 2002). Moreover, the observed $\mathrm{X} / K$ correlation implies that, for a given hard $\mathrm{X}$-ray flux, the $K$ magnitude can be predicted reasonably well $(\sigma \sim 1.3 \mathrm{mag})$ with a single relation both for broad line and ERO AGN.

\footnotetext{
5 The $R$-band flux is computed by converting $R$ magnitudes into monochromatic fluxes and then multiplying them by the width of the $R$ filter (Zombeck 1990). For a given X-ray energy range and $R$-band magnitude the following relation holds: $\log (\mathrm{X} / O)=\log f_{\mathrm{X}}+R / 2.5+$ const. where $f_{\mathrm{X}}$ is the $\mathrm{X}$-ray flux, $R$ is the optical magnitude and const. depends only on the $R$-band filter used in the optical observations; an indicative, average value is const. $=5.5$ (see Hornschemeier et al. 2000) and it can be used when datasets from different observations are compared.
}

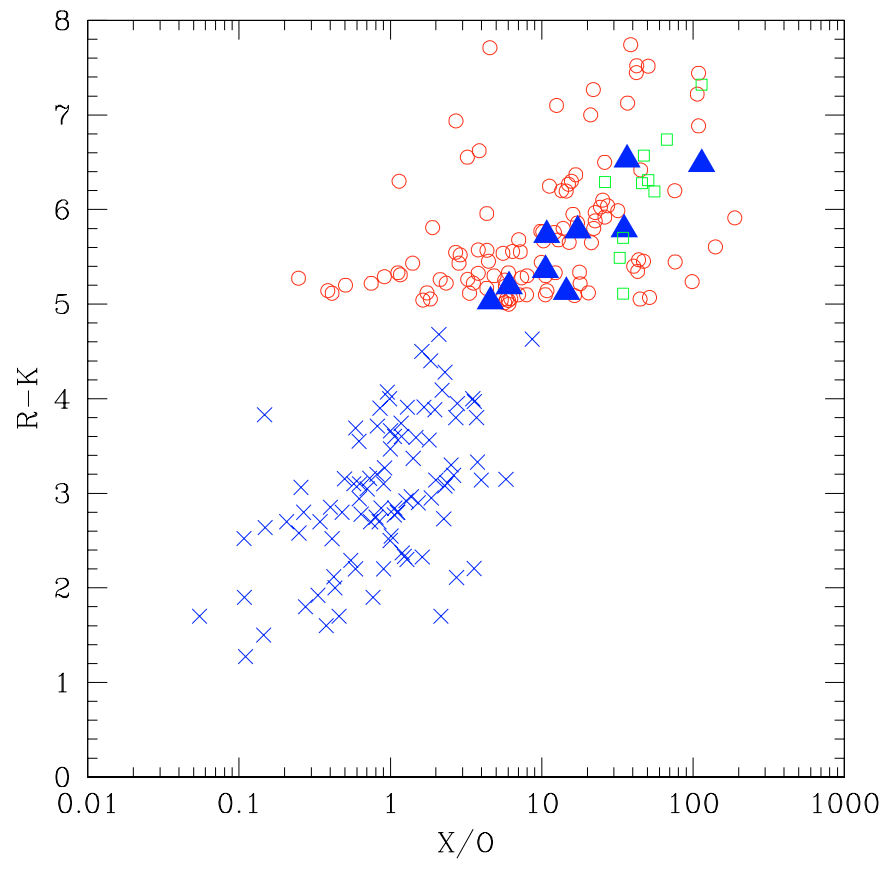

Fig. 5. The $R-K$ color as a function of the X-ray to optical flux ratio $(\mathrm{X} / O)$ for EROs in the literature sample (empty circles are objects from the deep fields; filled triangles the EROs from this work; empty squares sources from Mignoli et al. 2004) and for BL AGN selected in the CDF-N and CDF-S fields (crosses). See text for details.

The relationship between the hard X-ray and near-infrared fluxes can also explain the trend observed between the $R-$ $K$ colors and the $\mathrm{X} / O$ for all the EROs in the literature sample compared with that observed for BL AGN (Fig. 5): the higher the $\mathrm{X} / O$ is, the redder the source.

\subsection{AGN in K-selected ERO samples}

It has been already pointed out, on the basis of a handful of isolated cases, that the optical and near infrared properties of EROs hosting an AGN are indistinguishable from the overall ERO population both from a spectroscopic and photometric point of view (Brusa et al. 2002; Cimatti et al. 2003; Mignoli et al. 2004). This seems to apply also to the average redshifts and absolute luminosities of AGN EROs when compared to the general ERO population. Figure 6 (upper panel) shows the $K-z$ plane for the 62 AGN EROs with redshift information (26 spectroscopic and 36 photometric) discussed in the present work compared with all the EROs detected in a $K$-selected survey, the K20 survey (Cimatti et al. 2003), for which spectroscopic or reliable photometric redshifts are available. Although this figure shows objects from samples with different $K \mathrm{~s} \mathrm{lim}$ its, it allows us to conclude that X-ray detected EROs appear to be largely indistinguishable from the general non-AGN EROs population, both classes being brighter than typical local $(z=0) L_{K}^{*}$ galaxies (Cole et al. 2001; continuous line) and, on average, similar to evolved $L_{K}^{*}$ galaxies at $z=1-1.5$ (Pozzetti et al. 2003; dashed line), and spanning on average a similar range in the redshift distribution $(z=0.7 \div 2.5)$. 

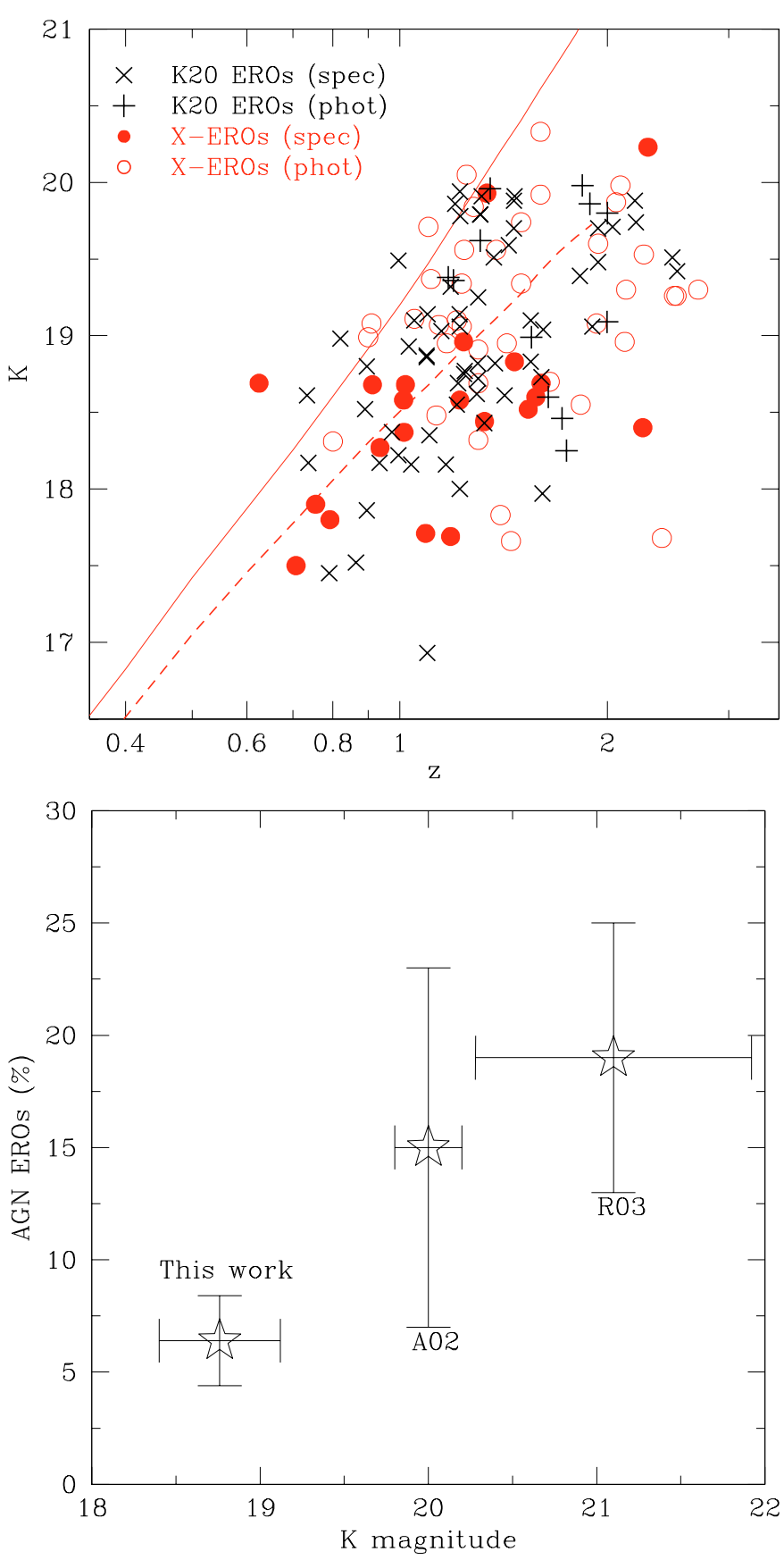

Fig. 6. Upper panel: the $K$ magnitude as a function of redshift for EROs in the literature sample for which spectroscopic (filled circles) or photometric (empty circles) redshifts are available and for nearinfrared selected EROs in the K20 survey (crosses: spectroscopic z; plus signs: photometric z). Lower panel: fraction of AGN ERO as a function of the $K$-band magnitudes, as computed from three different samples: this work, the Alexander et al. (2002) work (A02) and the Roche et al. (2003) sample.

On the basis of the considerations above, we can conclude that the X-ray emission can be considered the only reliable tracer of AGN activity in the EROs population, for which an accurate analysis at longer wavelengths is generally hampered by the faintness of the optical counterparts. Therefore, assuming that the observed distribution of the $\mathrm{X} / K$ ratios $(\mathrm{X} / K=0.1-10$, see Fig. 4) is representative of the AGN EROs population, it is possible to derive an estimate of the fraction of AGN EROs among $K$-selected samples, as a function of the $K$-band magnitude only and independent from the X-ray limiting fluxes. Indeed, given that the ratio between the $2-10 \mathrm{keV}$ and $K$ s limiting fluxes in our XMM-Newton sample is $\mathrm{X} / K \sim 1$, only about half of the shaded area in the lower panel of Fig. 4 is sampled at $K \sim 19$, and a bias against sources brighter than $K \mathrm{~s} \sim 19$ and with $F_{2-10 \mathrm{keV}} \lesssim 4 \times 10^{-15} \mathrm{erg} \mathrm{cm}^{-2} \mathrm{~s}^{-1}$ is present. We have therefore "corrected" the estimated fraction of AGN EROs reported in Sect. 3 taking into account the observed distribution of the X-ray to near-infrared ratios: the fraction of AGN EROs at $K \sim 19$ rises to $7 \pm 2 \%$. In the same way, it is possible to statistically "correct" the observed values in the A02 and R03 samples already reported in Sect. 3, in order to derive the AGN fraction at $K=20.1$ and $K=21.5$, respectively. At the limiting fluxes of the A02 sample, the $\mathrm{X} / K$ ratio is $\sim 0.2$, while at the R03 sample limiting fluxes, the observed $\mathrm{X} / K$ is $\sim 1.5$. Combining the depth of the two different samples with the observed X-ray to optical flux ratios distribution, the estimates of the fraction of AGN among EROs in the A02 and R03 samples rise to $(15 \pm 8) \%$ and $(19 \pm 6 \%)$, respectively.

In the lower panel of Fig. 6 these fractions for the three samples are shown at representative $K$-band magnitudes. Even if the statistical error bars are large, Fig. 6 suggests that the fraction of AGN EROs among the $K$-selected EROs population is an increasing function of the $K$-band magnitude. The results from hard X-ray surveys indicate a space density of low-luminosity $\left(10^{42}-10^{44} \mathrm{erg} \mathrm{s}^{-1}\right)$ AGN almost two orders of magnitude higher than that of high luminosity sources (Fiore et al. 2003; Ueda et al. 2003). Thus, it is not surprising that the fraction of AGN EROs increases going toward faint fluxes (i.e., lower luminosities). The fraction of "active" objects in $K$-selected EROs samples can be used to constrain models which link the formation and evolution of galaxies and AGN (e.g. Granato et al. 2004).

\section{X-ray properties of AGN EROs}

To check whether X-ray absorption is common among hard $\mathrm{X}$-ray detected EROs, we have quantitatively measured the intrinsic X-ray column densities for the 62 EROs with a spectroscopic or photometric redshift available in the literature sample described in Sect. 4.1.

Column densities for the sources detected in the CDF-N and $\mathrm{CDF}-\mathrm{S}$ have been obtained by fitting the observed counts with a single power law model plus absorption at the source redshift. When the quality of the X-ray spectra in terms of S/N ratio was not sufficient to use the standard $\chi^{2}$ statistic (a limit of 150 counts over the $0.5-8 \mathrm{keV}$ band has been assumed), the C-Statistic was used (Cash 1979). In this case the power-law spectral index has been fixed at $\Gamma=1.9$. For the sources from the Lockman Hole, the Hellas2Xmm and the "additional" sample, the best-fit values quoted by the authors have been adopted. In all the cases, $2-10 \mathrm{keV}$ luminosities were estimated from the observed X-ray fluxes and corrected for absorption.

The results are reported in Fig. 7. Almost all of the individually detected EROs are consistent with intrinsic column densities in excess of $10^{22} \mathrm{~cm}^{-2}$, and they actually are 
heavily obscured AGN. This study statistically confirms previous evidences some of which are based on HR analysis (Alexander et al. 2002) and on spectral analysis (e.g. Vignali et al. 2003; Gandhi et al. 2004; Willott et al. 2003; Stevens et al. 2003; Severgnini et al. 2005), and unambiguously indicates that large columns of cold gas (even $>10^{23} \mathrm{~cm}^{-2}$ ) are the rule rather than the exception in EROs individually detected in X-rays.

\subsection{EROs and QSO2: a selection criterion}

Given the high redshift $(z \gtrsim 1)$ and the X-ray flux of these objects, it follows that the majority of X-ray detected EROs have high X-ray luminosities $\left(L_{X}>10^{43} \mathrm{erg} \mathrm{s}^{-1}\right.$, see Fig. 7). Moreover, according to our analysis, a large fraction of the objects analysed in this work for which redshift information is available have X-ray luminosities even larger than $10^{44} \mathrm{erg} \mathrm{s}^{-1}$, and therefore well within the quasar regime. The large intrinsic column densities further imply that AGN EROs, selected at the brightest X-ray fluxes, have properties similar to those of type 2 quasars (QSO2), the high-luminosity, high redshift type II AGNs predicted by X-ray background synthesis models and necessary to reproduce the $2-10 \mathrm{keV}$ source counts at relatively bright fluxes (e.g. Comastri et al. 2001; Gilli et al. 2001).

On the basis of unified schemes, type II quasars are expected to be luminous, narrow-line, high-redshift objects with substantial $\left(N_{\mathrm{H}}>10^{22} \mathrm{~cm}^{-2}\right)$ X-ray column densities. However, at the faint fluxes/magnitudes of deep surveys the optical identifications of these objects is very difficult, unless one of the strong emission lines is present in the optical spectrum. Our analysis suggests that an efficient method to pick up this elusive population is the combination of medium-deep X-ray observations and $K$-band imaging: among hard $\mathrm{X}$-ray sources, one must select those counterparts with an $R-K>5$ color - that is an indication of high redshift and obscuration - and with an $\mathrm{X} / O$ ratio $>10$ - that is an indication of high column densities and high luminosity (see also Severgnini et al. 2005; Gandhi et al. 2004). This is shown in Fig. 7, where EROs with $\mathrm{X} / O>10$ are reported as filled symbols and populate the upper right region of the diagram (the "QSO2 locus"). The present work therefore confirms that a selection on the basis of $\mathrm{X} / O>10$ is a powerful tool to detect high-luminosity, highly obscured sources as already pointed out by Fiore et al. (2003), and it is even stronger when coupled with a previous selection on the basis of extremely red colors.

It is important to stress that EROs with high $\mathrm{X} / O$ can be QSO2, but it is not true that all the QSO2 are EROs. As an example, the prototype of high-redshift QSO2, CDFS_202 in Norman et al. (2002), has $R-K \sim 2.5$. In this case the rather blue observed colour is mainly due to the presence of a strong emission line in the $R$ filter. When the line flux is subtracted, the $R-K$ colour of CDFS_202 is $R-K \sim 4$, which makes this object appreciably red.

The close link between X-ray bright EROs and type 2 quasars allows us to elaborate on the contribution of EROs to the population of high luminosity, highly obscured

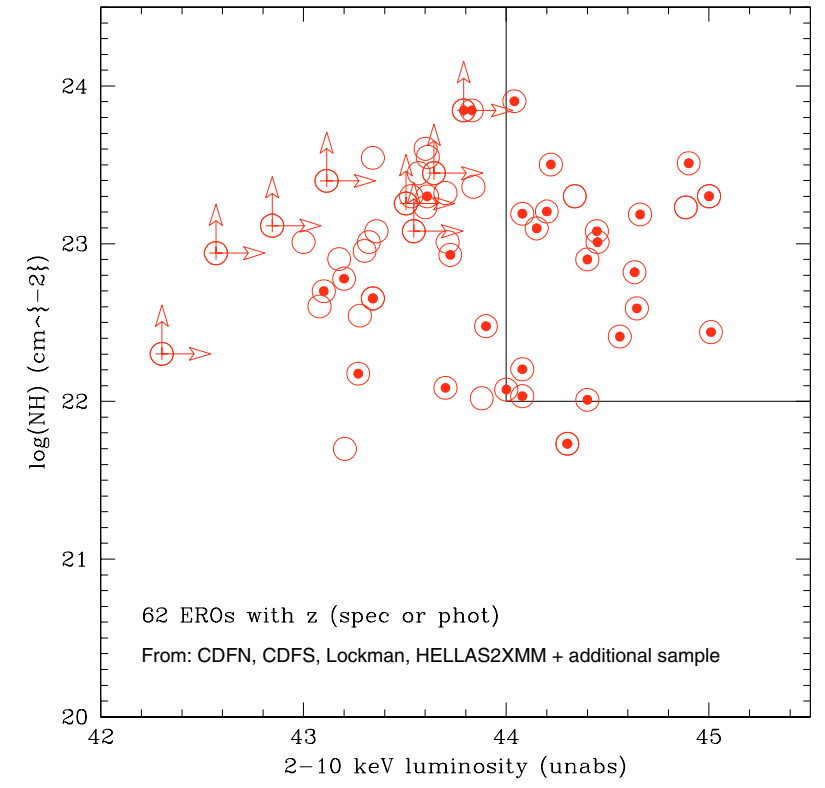

Fig. 7. Logarithm of the absorbing column density $\left(N_{\mathrm{H}}\right)$ versus the logarithm of the unabsorbed X-ray luminosity in the $2-10 \mathrm{keV}$ band for all the X-ray detected EROs with spectroscopic or photometric redshifts from the literature sample. Filled symbols are those with $\mathrm{X} / O>10$ (see text). In the upper right corner the "QSO2 locus" is highlighted.

quasars. We have considered only the sources with redshift information detected in the CDFN, CDFS, and in the Lockman Hole. In addition, using the method extensively discussed by Fiore et al. (2003) based on the relation between the $\mathrm{X} / O$ and the X-ray luminosity, we have derived the redshifts and luminosities also for the 9 hard X-ray detected EROs in our XMM-Newton observation. All but two have unabsorbed $\mathrm{X}$-ray luminosities larger than $10^{44} \mathrm{erg} \mathrm{s}^{-1}$ and lie in the redshift range $z=1-3$.

To estimate the contribution of EROs to the QSO2 population we have chosen a $2-10 \mathrm{keV}$ limiting flux of $\sim 10^{-14} \mathrm{erg} \mathrm{cm}^{-2} \mathrm{~s}^{-1}$, in order to ensure a flat and uniform sky coverage down to fluxes where about half of the XRB flux is resolved. The most recent published determinations of the space density of type 2 quasars at fluxes brighter than this limit are in the range $40-50 \mathrm{deg}^{2}$ (Perola et al. 2004; Padovani et al. 2004) while the prediction from the Ueda et al. (2003) model is $\sim 75 \mathrm{deg}^{-2}$ (private communication). From our analysis, a total of 6 EROs over an area of about $0.4 \mathrm{deg}^{2}$ have been detected at fluxes $\gtrsim 10^{-14} \mathrm{erg} \mathrm{cm}^{-2} \mathrm{~s}^{-1}$ and classified as QSO2, i.e. they have $N_{\mathrm{H}}>10^{22} \mathrm{~cm}^{-2}$ and unabsorbed $L_{2-10 \mathrm{keV}}>10^{44} \mathrm{erg} \mathrm{s}^{-1}$. Therefore, the surface density of luminous, obscured EROs is about $15 \mathrm{deg}^{-2}$, and it has to be regarded as a robust lower limit given the lack of redshift information for some of the EROs in the present sample. This work therefore indicates that AGN EROs represent at least $20 \%$ of the type 2 quasars population, if compared to the Ueda et al. (2003) predictions, and it can be as high as $\sim 40 \%$ when compared with the recently published estimates (Padovani et al. 2004; Perola et al. 2004). 


\section{2. $X / K$ correlation and the accretion parameters of AGN EROs}

While there is a fairly weak correlation between the X-ray flux and the optical magnitude (upper panel of Fig. 4), a linear correlation with a smaller scatter appears to hold between the Xray and $K$-band fluxes (right panel of Fig. 4). This relation is present despite the large redshift range (e.g. $z \simeq 0-3$ ) of our sources and may reflect a proportionality also between the luminosities, $L_{\mathrm{X}} \propto L_{K}$. Such a correlation is reminiscent of the ones observed locally between the $\mathrm{BH}$ mass and the global galaxy properties (Magorrian et al. 1998; Gebhardt et al. 2000; Ferrarese \& Merritt 2000; Marconi \& Hunt 2003) and may be related to them.

In order to test such a possibility with a conservative approach we have considered those EROs with a secure spectroscopic identification in the comparison sample and the 9 EROs in our XMM-Newton observation, using the Fiore et al. (2003) relation to estimate their redshifts. Although EROs and bluer AGN have a similar $\mathrm{X} / K$ ratio, a result taht is surprising given that both the active nucleus and the host galaxies are contributing to the $K$-band light presumably in different ratios, we limit our analysis to EROs since there are several indications that the near-infrared emission of these X-ray selected obscured AGN is dominated by their host galaxy starlight (see e.g. Mainieri et al. 2002; Mignoli et al. 2004). The rest-frame $K$-band luminosities have been computed using an evolving elliptical galaxy template to properly account for the K-corrections ${ }^{6}$ (Bruzual \& Charlot 2003). The results are shown in Fig. 8.

The correlation between the near-infrared and X-ray luminosities observed for these high-redshift EROs (i.e. $L_{\mathrm{X}} \propto L_{K}$ ) closely resembles the one published by Marconi \& Hunt (2003) between the BH mass and the $K$ band luminosity for a sample of local galaxies. Assuming that the X-ray luminosity of our AGN EROs is proportional to the $\mathrm{BH}$ mass (i.e. the Eddington ratio $L / L_{\mathrm{Edd}}$ and the bolometric correction $k_{\mathrm{bol}}{ }^{7}$ are not a strong function of the $\mathrm{BH}$ mass), the observed correlation plotted in Fig. 8 implies $L_{\mathrm{K}} \propto M_{\mathrm{BH}}$. It is thus formally possible to tentatively constrain the $\mathrm{BH}$ masses and the accretion parameters that would follow if the normalization of such a relation does not significantly evolve with redshift, apart from the expected change of stellar mass to light ratio of the host galaxies due to the evolution of the stellar populations (our AGN EROs sample being at $z=1-2$ ). The resulting $M_{\mathrm{BH}}$ are reported on the right axis of Fig. 8. The two continuous lines in Fig. 8 represent the relation between the $\mathrm{BH}$ mass and X-ray luminosity computed for Eddington-limited accretion $\left(L_{\mathrm{bol}} / L_{\mathrm{edd}}=1\right)$ and for two different values $\left(k_{\mathrm{bol}}=30\right.$ and $\left.k_{\mathrm{bol}}=10\right)$ of the bolometric correction. The first value is from the Quasar SED compilation of Elvis et al. (1994) and can be considered a reliable correction for bright unobscured quasars. The second appears to be appropriate for lower luminosities $\left(L_{\mathrm{X}} \sim 10^{43}-10^{44} \mathrm{erg} \mathrm{s}^{-1}\right)$ Seyfert-like galaxies (Fabian 2004) and a few heavily obscured,

\footnotetext{
${ }^{6}$ Note that in the $K$-band the $\mathrm{K}$-corrections are relatively insensitive to galaxy type and are fairly small up to $z \lesssim 2$.

7 The absorption-corrected X-ray luminosity can be translated into a bolometric luminosity assuming a bolometric correction factor $\left(L_{\mathrm{bol}}=k_{\mathrm{bol}} \times L_{\mathrm{X}}\right)$.
}

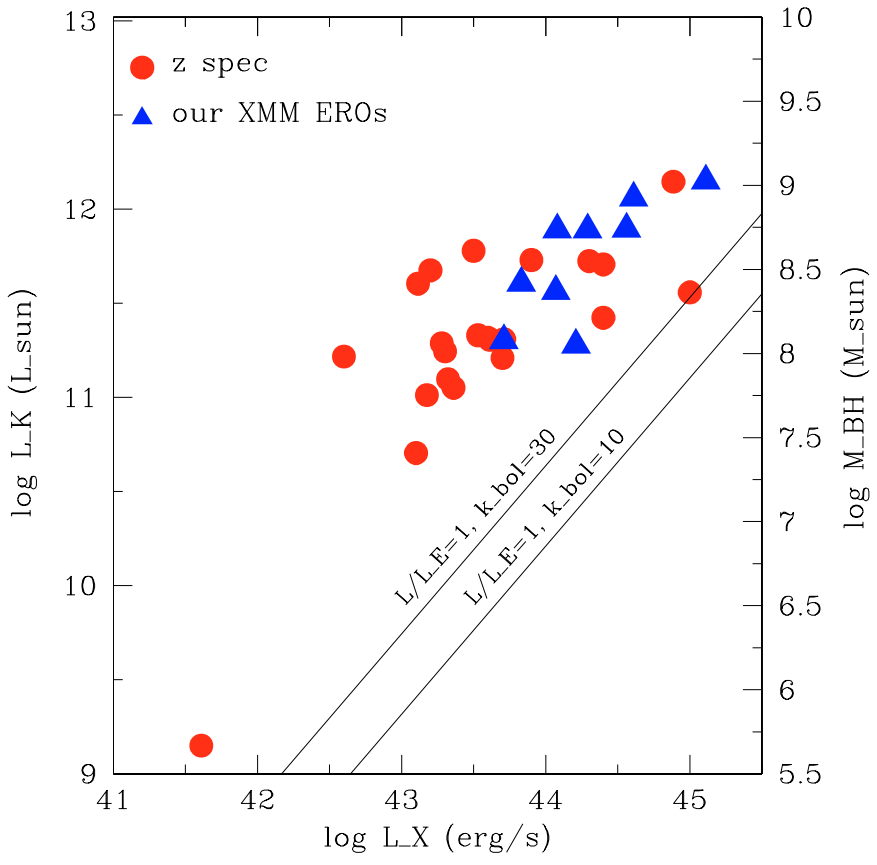

Fig. 8. $K$-band luminosities versus the $2-10 \mathrm{keV} X$-ray luminosities (filled triangles: our XMM EROs; filled circles: EROs with spectroscopic redshifts). The $M_{\mathrm{BH}}$ resulting from the $K$-band luminosity on the basis of the Marconi \& Hunt (2003) relation are reported on the right axis of the figure. The two continuous lines represent the expected correlation between the two plotted quantities for two different assumptions on the bolometric correction $\left(k_{\mathrm{bol}}=10\right.$ and $\left.k_{\mathrm{bol}}=30\right)$ and for $L / L_{\mathrm{Edd}}=1$.

luminous sources (Comastri 2004). The value observed for our objects can be reasonably well explained by a spread in the Eddington ratios in the range $L_{\mathrm{bol}} / L_{\mathrm{Edd}}=2 \times 10^{-3} \div 1$, with a median value of $L_{\mathrm{bol}} / L_{\mathrm{Edd}}=0.03-0.1$ for $k_{\mathrm{bol}}=10$ and 30 , respectively. Both the $\mathrm{BH}$ masses and the Eddington ratios derived above are consistent with a scenario in which X-ray detected EROs are obscured quasars emitting in a radiatively efficient way, in agreement with the results of Merloni (2004) and McLure \& Dunlop (2004). These findings are broadly consistent with those obtained, with completely different methods, by Woo \& Urry (2002) for a large sample of broad line AGN (see their Fig. 8).

Although the discussion above has been conducted only in a qualitative way and neglecting the uncertainties and scatter associated with the observed relations, it appears reasonable that the close correlation that we have detected between the $\mathrm{X}$-ray flux and the $K$-band magnitude of AGN EROs is the high redshift analogous to the correlations observed locally, implying a close connection between the black hole and AGN host galaxies also at earlier epochs $(z=1-2)$.

\section{Summary}

In this paper we have presented the results from an $80 \mathrm{ks}$ XMM-Newton observation of the largest sample of nearinfrared selected EROs available to date down to a $K$-band magnitude limit of $K=19.2$ (Daddi et al. 2000). The moderate-deep exposure and the high energy throughput of 
XMM-Newton, coupled with its large field of view, allowed us to detect, for the first time on a statistically significant sample, AGN-powered EROs at relatively bright X-ray fluxes. At the limiting fluxes probed by our survey $\left(F_{2-10 \mathrm{keV}} \gtrsim 4 \times 10^{-15}\right.$ and $K \mathrm{~s} \lesssim 19.2)$ the fraction of AGN EROs within near-infrared selected ERO samples is $\sim 3.5 \%$. Conversely, a significant fraction of the optical counterparts of hard X-ray selected sources are EROs and the fraction of extremely red objects among the $\mathrm{X}$-ray population is much higher $(\sim 20 \%)$.

The average hardness ratio of the hard X-ray detected EROs in the XMM-Newton observation suggest substantial column densities at the source redshift.

In order to place our results in a broader context, we have also considered additional samples of X-ray detected EROs available in the literature from published deep and medium deep hard X-ray surveys. A total of 128 X-ray detected EROs have been considered, and for the first time the average $\mathrm{X}$-ray, optical and near-infrared properties of AGN-powered EROs have been derived on a statistically significant sample. The most important results of our analysis are summarized in the following:

- The average X-ray to optical flux ratio of AGN EROs is about one order of magnitude higher than that observed for BL AGN. On the contrary, when the X-ray to near-infrared properties are considered, all the EROs in the comparison sample occupy a locus which is indistinguishable from that occupied by unobscured QSO.This result further corroborates the hypothesis that AGN EROs are obscured quasars.

- High obscuration in X-ray detected EROs is also revealed in the X-ray band, in agreement with the results from the optical band: the majority of the sources with known redshifts of the comparison sample have $N_{\mathrm{H}}>10^{22} \mathrm{~cm}^{-2}$, and about half are in excess of $10^{23} \mathrm{~cm}^{-2}$. The observed $\mathrm{X}$-ray fluxes and spectral shapes imply unabsorbed, rest frame X-ray luminosities in the range of $10^{42}-10^{45} \mathrm{erg} \mathrm{s}^{-1}$. At fluxes larger than $10^{-14} \mathrm{erg} \mathrm{cm}^{-2} \mathrm{~s}^{-1}$, we estimate that AGN EROs contribute at least $20 \%$ (and possibly up to $40 \%$ ) to the QSO2 population. A selection criterion based on the X/O and the $R-K$ colour of hard X-ray selected sources has been proposed to efficiently detect this elusive population of highly obscured quasars that are one of the key ingredients of XRB synthesis models.

- Assuming that the observed $\mathrm{X} / K$ distribution of EROs is representative of the AGN ERO population, and combining our results with those of A02 and R03, we found evidence of an increase in the fraction of AGN EROs in $K$-selected EROs samples as a function of the $K$-band magnitude, ranging from $\sim 6 \%$ at $K=19$ to $\sim 20 \%$ at $K=22$.

- The close relation uncovered between the $K$ - and X-ray band fluxes of AGN EROs suggests that the connection between the properties of the host galaxies and central black holes observed locally holds also at higher redshifts $(z \simeq 1-2)$. This allows an estimate of the $\mathrm{BH}$ masses and Eddington ratios for the sources with known redshift through reasonable assumptions. The results indicate that the majority of AGN-powered EROs have $\mathrm{BH}$ masses larger than $5 \times 10^{7} M_{\odot}$, and are accreting with a median value of $L_{\mathrm{bol}} / L_{\mathrm{Edd}} \sim 0.03-0.1$.

All the findings discussed above support the idea that hard X-ray surveys coupled with near-infrared observations provide an efficient method to detect QSO2. Furthermore, X-ray detected EROs can be used as tools to investigate the accretion paradigm at high redshifts and to address the issue of elliptical galaxy formation and the expected co-evolution with accreting black-holes. In particular, systematic studies of the relationship between EROs and QSO2 are needed to quantitatively investigate the link between the formation of massive elliptical galaxies and the onset of AGN activity.

$\mathrm{X}$-ray observations of large samples of $K$-selected EROs would be crucial to compute the fraction of X-ray active EROs over the widest area possible (to avoid cosmic variance). Conversely, deep optical and near-infrared follow-up of complete samples of hard X-ray selected sources with extreme $\mathrm{X} / O$ will help determine the fraction of reddened sources among the XRB constituents. The full exploitation of the COSMOS multiwavelength database in the near future will be the best strategy to investigate both these issues. The large area covered $\left(\sim 2 \mathrm{deg}^{2}\right)$ will allow a detailed study of the clustering properties of these objects and could shed new light on the link between nuclear activity and galaxy evolution.

Acknowledgements. We gratefully acknowledge the K20 team for providing their data, and Y. Ueda for providing his model predictions. M.B. and A.C. acknowledge support by INAOE, Mexico, during the 2003 Guillermo-Haro Workshop where part of this work was performed. MB acknowledges partial support from the Deutscher Akademischer Austausch Dienst (DAAD, German Academic Exchange Service) under the project: "New Frontiers in Science". The XMM-Newton Helpdesk and the entire XMM-Newton team, in particular B. Altieri, M. Guainazzi and G. Vacanti, are kindly acknowledged for their help with astrometry problems. The authors acknowledge partial support by ASI I/R/057/02 and MIUR COFIN03-02-23 contracts, and INAF 270/2003 grant.

\section{References}

Alexander, D. M., Brandt, W. N., Hornschemeier, A. E., et al. 2001, AJ, 122, 2156

Alexander, D. M., Vignali, C., Bauer, F. E., et al. 2002, AJ, 123, 1149 (A02)

Baldi, A., Molendi, S., Comastri, A., et al. 2002, ApJ, 564, 190

Barger, A. J., Cowie, L. L., Capak, P., et al. 2003, AJ, 126, 632

Brusa, M., Comastri, A., Daddi, E., et al. 2002, ApJ, 581, L89

Brusa, M. 2003, AN, 324, 116

Brusa, M., Comastri, A., Mignoli, M., et al. 2003, A\&A, 409, 65

Bruzual, A. G., \& Charlot, S. 2003, MNRAS, 344, 1000

Cash, W. 1979, ApJ, 228, 939

Ciliegi, P., Zamorani, G., Hasinger, G., et al. 2003, A\&A, 398, 901

Cimatti, A., Daddi, E., Mignoli, M., et al. 2002, A\&A, 381, L68

Cimatti, A., Daddi, E., Cassata, P., et al. 2003, A\&A, 412, L1

Cole, S., Norberg, P., Baugh, C. M., et al. 2001, MNRAS, 326, 255

Comastri, A., Fiore, F., Vignali, C., et al. 2001, MNRAS, 327, 781

Comastri, A., Brusa, M., \& Mignoli, M. 2003, AN, 324, 28

Comastri, A. 2004, in Supermassive Black Holes in the Distant Universe, ed. A. J. Barger (Boston: Kluwer Academic Publishers), 308,245 
Crawford, C. S., Gandhi, P., Fabian, A. C., et al. 2002, MNRAS, 333, 809

Daddi, E., Cimatti, A., Pozzetti, L., et al. 2000, A\&A, 361, 535

Dickey, J. M., \& Lockman, F. J. 1990, ARA\&A, 28, 215

Elston, R., Rieke, G. H., \& Rieke, M. J. 1988, ApJ, 331, L77

Elvis, M., Wilkes, B. J., McDowell, J. C., et al. 1994, ApJS, 95, 1

Fabian, A. C. 2004, in Coevolution of Black Holes and Galaxies, ed. L. C. Ho (Cambridge Univ. Press), Carnegie Observatories Astrophysics Series, 1, 447

Ferrarese, L., \& Merritt, D. 2000, ApJ, 539, L9

Fiore, F., Brusa, M., Cocchia, F., et al. 2003, A\&A, 409, 79

Franceschini, A., Hasinger, G., Miyaji, T., \& Malguori, D. 1999, MNRAS, 310, L5

Gandhi, P., Crawford, C. S., Fabian, A. C., \& Johnstone, R. M. 2004, MNRAS, 348, 529

Gebhardt, K., Bender, R., Bower, G., et al. 2000, ApJ, 539, L13

Gilli, R., Salvati, M., \& Hasinger, G. 2001, A\&A, 366, 407

Granato, G. L., Silva, L., Monaco, P., et al. 2001, MNRAS, 324, 757

Granato, G. L., De Zotti, G., Silva, L., Bressan, A., \& Danese, L. 2004, ApJ, 600, 580

Hall, P. B., Yee, H. K. C., Lin, H., et al. 2000, AJ, 120, 2220

Hasinger, G., Giacconi, R., Gunn, J. E., et al. 1998, A\&A, 340, L27

Hornschemeier, A. E., Brandt, W. N., Garmire, G. P., et al. 2000, ApJ, 541,49

Jansen, F., Lumb, D., Altieri, B., et al. 2001, A\&A, 365, L1

Kauffmann, G. 2004, in Star Formation Through Time, ed. E. Perez, R. M. Gonzalez Delgado, \& G. Tenorio-Tagle, ASP Conf. Proc., 297, 439

Koekemoer, A. M., Alexander, D. M., Bauer, F. E., et al. 2004, ApJ, 600, L123

Lehmann, I., Hasinger, G., Schmidt, M., et al. 2001, A\&A, 371, 833

Lehmann, I., Hasinge, R. G., Murray, S. S., \& Schmidt, M. 2002, in Proc. of X-rays at Sharp Focus Chandra Science Symposium, ASP Conf. Proc., 262, 105

Magorrian, J., Tremaine, S., Richstore, D., et al. 1998, AJ, 115, 2285

Mainieri, V., Bergeron, J., Hasinger, G., et al. 2002, A\&A, 393, 425

Mannucci, F., Pozzetti, L., Thompson, D., et al. 2002, MNRAS, 329, L57
Marconi, A., \& Hunt, L. 2003, ApJ, 589, L21

Marconi, A., Risaliti, G., Gilli, R., et al. 2004, MNRAS, 351, 169

McLure, R. J., \& Dunlop, J. S. 2004, MNRAS, 352, 1390

Menci, N., Fiore, F., Perola, G. C., \& Cavaliere 2004, ApJ, 606, 58

Merloni, A. 2004, MNRAS, 353, 1035

Mignoli, M., Pozzetti, L., Comastri, A., et al. 2004, A\&A, 418, 827

Miyazaki, M., Shimasaku, K., Kodama, T., et al. 2003, PASJ, 55, 1079

Norman, C., Hasinger, G., Giacconi, R., et al. 2002, ApJ, 571, 218

Padovani, P., Allean, M. G., Rosati, P., \& Walton, N. A. 2004, A\&A, 424, 545

Perola, G. C., Puccetti, S., Fiore, F., et al. 2004, A\&A, 421, 491

Pierre, M., Lidman, C., Hunstead, R., et al. 2001, A\&A, 372, L45

Pozzetti, L., \& Madau, P. 2000, in The Extragalactic Infrared Background and its Cosmological Implications, ed. M. Harwitt, \& M. G. Hauser, IAU Symp., 204, 71

Pozzetti, L., Cimatti, A., Zamorani, G., et al. 2003, A\&A, 402, 837

Roche, N., Dunlop, J., \& Almaini, O. 2003, MNRAS, 346, 803 (R03)

Rosati, P., Tozzi, P., Giacconi, R., et al. 2002, ApJ, 566, 667

Severgnini, P., Della Ceca, R., Braito, V., et al. 2003, Proc. of the Venice conference, Multiwavelength Mapping of Galaxy Formation and Evolution [arXiv: astro-ph/0312098]

Severgnini, P., Della Ceca, R., Braito, V., et al. 2005, A\&A, 431, 87

Smail, I., Owen, F. N., Morrison, G. E., et al. 2002, ApJ, 581, 844

Stevens, J. A., Page, M. J., Ivison, R. J., et al. 2003, MNRAS, 342, 249

Sutherland, W., \& Saunders, W. 1992, MNRAS, 259, 413

Szokoly, G. P., Bergeron, J., Hasinger, G., et al. 2004, ApJS, 155, 271

Thompson, D., Beckwith, S. V. W., Fockenbrock, R., et al. 1999, ApJ, 523,100

Tozzi, P., Rosati, P., Nonino, M., et al. 2001, ApJ, 562, 42

Ueda, Y., Akiyama, M., Ohta, K., \& Miyaji, T. 2003, ApJ, 598, 886

Vignali, C., Alexander, D. M., Bauer, F. E., et al. 2002, Proc. of the 5th Italian AGN Meeting, Inflows, Outflows and Reprocessing around black holes [arXiv: astro-ph/0209415]

Willott, C., Rawlings, S., Jarvis, M. J., \& Blundell, K. M. 2003, MNRAS, 339, 397

Woo, J. H., \& Urry, C. M. 2002, ApJ, 579, 530

Yan, L., Thompson, D., \& Soifer, B. T. 2004, AJ, 127, 1274 